\title{
An Algorithm for Optimization of Experimental Parameters for Maximum Uniformity of Film Thickness
}

\author{
B. S. Ramprasad, T. S. Radha, and M. Ramakrishna Rao \\ Central Instruments and Services Laboratory, Indian Institute of Science, Bangalore 12, India
}

\begin{abstract}
A simple method for evaluating the geometrical parameters yielding maximum uniformity of film thickness on rotating substrates is described. The algorithm is based on numerical integration of the function for relative thickness. It is shown that maximum uniformity is obtained for a normalized source-to-substrate distance ratio of $h / \delta=1.37$.
\end{abstract}

\section{Introduction}

Most applications of thin films require uniformity of thickness over the substrate area. The film thickness is predicted by equations based on the Knudsen's cosine law. These theoretical predictions in one form or another have been made use of by many workers ${ }^{1-5}$ for obtaining uniform films for different purposes. Uniformity is influenced by the geometrical parameters, that is, the position of the source with respect to the substrate, geometry of substrate and source, etc. Behrndt ${ }^{2}$ has verified the geometry for a particular case where thickness variations as small as $0.35 \%$ over a $4 \times 4$ in. substrate can be obtained. The objective of this investigation is to arrive at a solution for optimization of the parameters to yield maximum uniformity. The paper describes a method using numerical integration by generalized Simpson's rule of the relative film thickness with respect to normalized substrate radius. By minimizing the difference between the area under this function and that for constant thickness, the condition for optimum uniformity is determined. This method can be used for similar optimization problems. For a rotating substrate it is shown, theoretically, that maximum uniformity is achieved for a normalized source-to-substrate distance ratio of $h / \delta=1.37$.

\section{Method of Optimization}

The relative thickness distribution on a rotating planar substrate for a small area surface source is given by

$t / t_{0}=\left(1+a^{2}\right)^{2}\left(1+a^{2}+b^{2}\right) /\left[\left(1+a^{2}+b^{2}\right)^{2}-4 b^{2}\right]^{2}$,

where $t_{0}=$ thickness on the substrate when the source is on the axis of rotation; $t=$ thickness at the point under consideration; $a=h / \delta$, normalized source to substrate distance; $b=s / \delta$, normalized radius of the substrate; $h=$ distance between source and substrate; and $\delta=$ distance between the source and the axis of rotation.

Relative thickness has been computed for various values of $a$ and $b$ on the electronic computer and the results have been already reported. ${ }^{6}$ Figure 1 shows the profile for three values of $a$. It can be seen that the profile nearest $t / t_{0}=1$ is the optimum solution for a chosen range of $b . b$ determines the maximum substrate diameter over which the predicted uniformity is possible. Restating the problem, the condition for maximum uniformity is that area enclosed between the straight line $t / t_{0}=1$, and the function should be minimum.

It can be seen from Fig. 2 that this condition is achieved when the shaded area becomes minimum.

The area under the function $f(a, b)$,

$$
\int_{b=0}^{b=b_{1}} f(a, b) d b=\int_{b=0}^{b=b_{1}} \frac{\left(1+a^{2}\right)^{2}\left(1+a^{2}+b^{2}\right)}{\left[\left(1+a^{2}+b^{2}\right)^{2}-4 b^{2}\right]^{3}} d b
$$

bounded by $b=0-b_{1}$, is found by application of the generalized Simpson's rule, using the digital computer. The difference between area under $t / t_{0}=1$ and the computed area for $f(a, b)$ is found. The optimum solution is given by the one nearest to zero.

The integration has been carried out for $b=0-0.5$ for 10 intervals for different values of $a$ ranging from $a=1.2$ to 1.5 in steps of 0.005 . The result has been obtained up to the sixth decimal place. Figure 1 shows that the optimum solution exists for an $a$ value between 1.3 and 1.4. The area under $t / t_{0}=1$ and $b$ bounded by $0-0.5$ is 0.5 . Hence the difference is

$$
0.5-\int_{0}^{0.5} f(a, b) d b .
$$

This has been calculated.

Table I gives a few of the selected values from the computer output. Further the same programme was

TABle I. Difference in area for some values of $a$.

\begin{tabular}{cc}
\hline \hline$a$ & $\mathbf{0 . 5}-\int_{0}^{0.5} f(a, b) d b$ \\
\hline 1.300 & -0.002079 \\
1.365 & -0.000127 \\
1.370 & 0.000007 \\
1.375 & 0.000139 \\
\hline
\end{tabular}




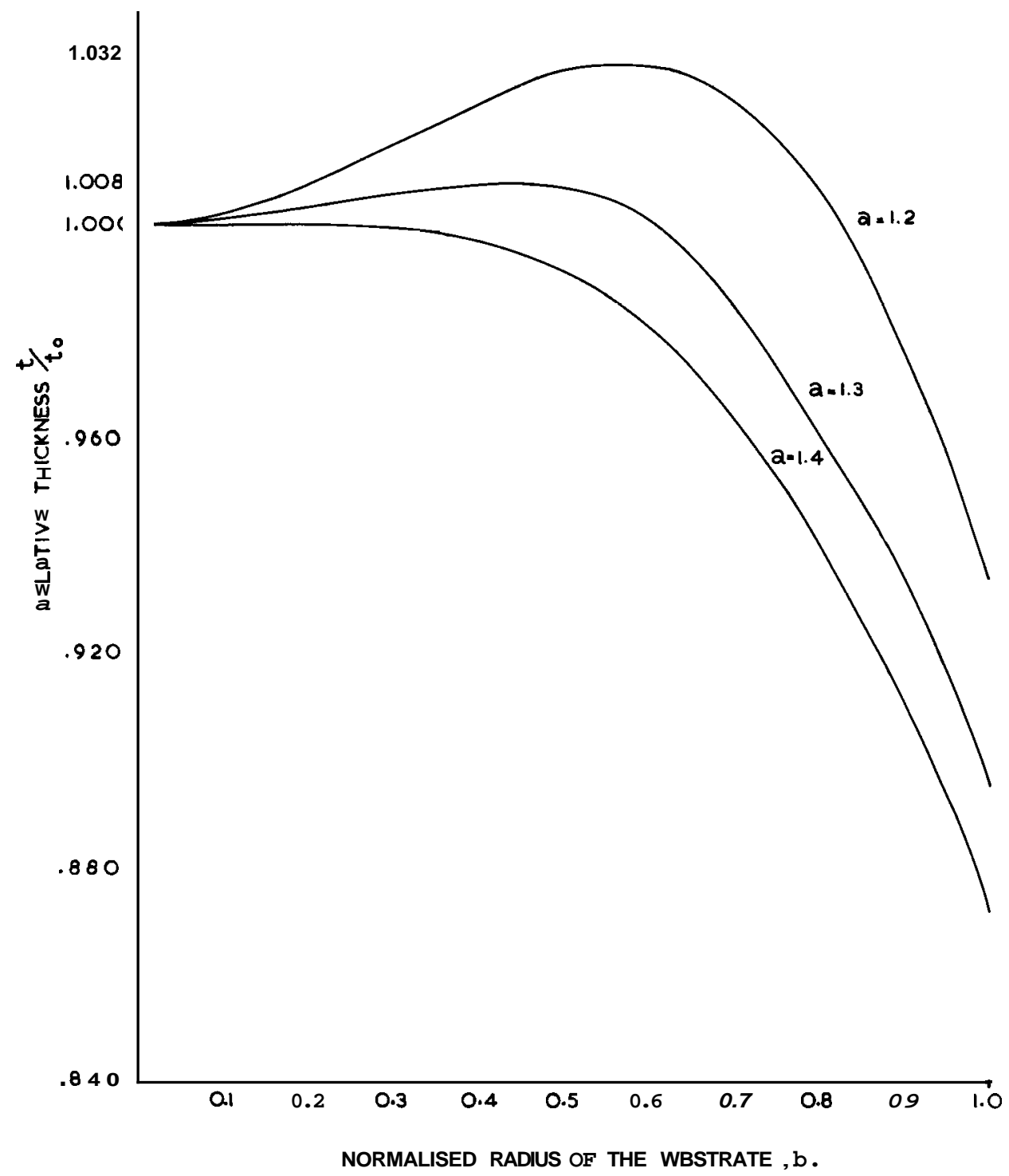

Figure 1. Relative thickness versus normalized substrate radius.

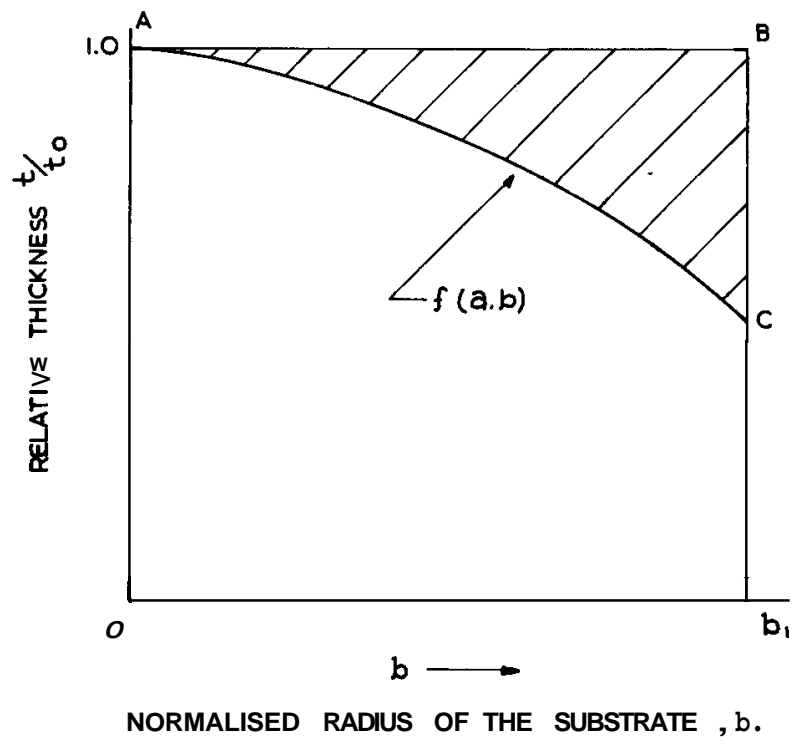

Figure 2. Condition for optimization. Shaded area $A B C$ to be minimized. run for $a$ ranging from $a=1.365$ to 1.370 in steps of .0005 . It is observed that for a value of 1.3695 , the difference is -0.000006 . It is noted here that the experimental value of 1.367 quoted by Behrndt ${ }^{2}$ for maximum uniformity of film thickness is very near the calculated value.

\section{Acknowledgment}

The authors gratefully acknowledge the assistance in computation rendered by S. K. Sen.

\section{References}

1. L. I. Maissel and R. Glang, Handbook of Thin Film Technology (McGraw-Hill, New York, 1970), pp. 55-59.

2. K. H. Behrndt, Transactions of the Tenth National Vacuum Symposium (Macmillan, New York, 1963), p. 379.

3. H. A. Macleod, Thin Film Optical Filters (Adam Hilger, London, 1969), p. 227.

4. P. H. Lissberger and J. Ring, Opt. Acta 2, 42 (1955).

5. B. S. Ramprasad, T. S. Radha, and M. Ramakrishna Rao, Indian J. Technol. 9, 8 (1971).

6. B. S. Ramprasad, T. S. Radha, and M. Ramakrishna Rao, J. Vac. Sci. Tcchnol. (to be published). 